\title{
The Active Cochlea
}

\author{
Peter Dallos \\ Auditory Physiology Laboratory (The Hugh Knowles Center) and Department of Neurobiology and Physiology, \\ Northwestern University, Evanston, Illinois 60208
}

The cochlea is a hydromechanical frequency analyzer located in the inner ear (Fig. 1a). Its principal role is to perform a realtime spectral decomposition of the acoustic signal in producing a spatial frequency map. The frequency analysis may be understood with the aid of Figure $1 b$, which shows a straightened cochlea with a snapshot of its basilar membrane displaced in response to a single-frequency sound (pure tone). Upon delivery of an acoustic signal into the fluid-filled cochlea, the basilar membrane undergoes an oscillatory motion at the frequency of the sound, resulting in a wave traveling toward its distal end. The drawing shows an instantaneous view of this traveling wave. The wave is spatially confined along the length of the basilar membrane, and the location of its maximum amplitude is related to the frequency of the sound. The higher the frequency, the more restricted the disturbance to the proximal end. The vibrating membrane supports the sense organ of hearing, the spiral organ of Corti (Fig. 2), which is deformed maximally in the region of the peak of the traveling wave. In this location, the sensory receptor cells of the organ of Corti receive maximal mechanical stimulation, transduce it into maximal electrical signals, and thus produce maximal afferent sensory outflow from the cochlea. Thus, mechanical frequency analysis is performed by matching particular frequencies with particular groups of auditory receptor cells and their ncrve fibcrs.

Understanding of frequency analysis in the inner ear progressed through three main epochs. The first was dominated by Helmholtz's suggestions that lightly damped, spatially ordered, mechanically resonant elements in the cochlea perform the spectral analysis (this period was reviewed by Wever, 1949). The second epoch, lasting from the late 1940 s to the early 1970 s, was dominated by von Békésy's description of the traveling wave (von Békésy, 1960). We are now in the third epoch (an overview of its evolution is given in Dallos, 1988), during which a fundamentally different paradigm has emerged. According to this paradigm, von Békésy's traveling wave is boosted by a local electromechanical amplification process in which one of the mammalian ear's sensory cell groups, outer hair cells, function as both sensors and mechanical feedback elements. The ideas that the operation of a sense organ is dependent upon local mechanical feedback modification by what resembles a sensory receptor cell, that such mechanical feedback may operate at audio frequencies utilizing some novel cellular motor, and that

This work was supported by NIDCD Grants DC00089 and DC00708.

Correspondence should be addressed to Peter Dallos at the above address.

Copyright (C) 1992 Society for Neuroscience $0270-6474 / 92 / 124575-11 \$ 05.00 / 0$ the resulting modification of the receptor output is responsible for the remarkable performance of the system, were certainly unexpected.

The biological processes that perform auditory frequency analysis vary among vertebrates: there is no such thing as the cochlear mechanism. One apparently widely used and also well understood mechanism among lower vertebrates is spectral analysis by electrical resonance in the sensory receptor cells themselves. In the turtle cochlea, these resonance frequencies range from 20 to $600 \mathrm{~Hz}$. This mechanism is reviewed by Fettiplace (1987). Mechanical resonance of the sensory cell's receptive organelles, the ciliary bundle, may be the principal means of frequency analysis in some other reptilian cochleae (Weiss et al., 1978).

In mammals, the hearing range generally extends to frequencies so high that simple resonance, electrical or mechanical, as used in many lower vertebrates, is no longer adequate for their analysis. Consequently, in mammals there is a shift away from analysis by the individual receptor cell as the tuned element, toward a concerted interaction among receptors and their greatly elaborated sensory accessory structures. The means of satisfying the demands upon the mammalian cochlea appear to be a nonlinear local feedback process, which is assumed to result in a cycle-by-cycle boost of vibratory amplitude (Gold, 1948; Davis, 1983). The amplification preferentially functions at low signal levels, and confers high sensitivity and wide dynamic and frequency range on the ear's operation.

Here, we consider cochlear function in mammalian ears only. Moreover, the discussion is restricted to the operation of the general mammalian ear, while specializations developed to serve infra- or ultrasonic hearing are not addressed.

\section{Design objectives}

Virtually all environmental and biologically significant sounds change their amplitude, frequency, and phase, from instant to instant. The ear must be capable of analyzing the time-varying features of these sounds and representing them in a spatiotemporal array of neural discharges in the fibers of the auditory nerve. When analyzing a signal, its frequency spectrum and tcmporal characteristics are reciprocally related. The spectrally simplest signal, a continuous sine wave, corresponding to a pure tone in acoustics, has only one spectral component, the frequency of the sound, and lasts forever. In contrast, the temporally simplest signal, an impulse, corresponding to a click in acoustics, is infinitesimally short in duration but contains all frequencies. Natural sounds present a continuum between these 

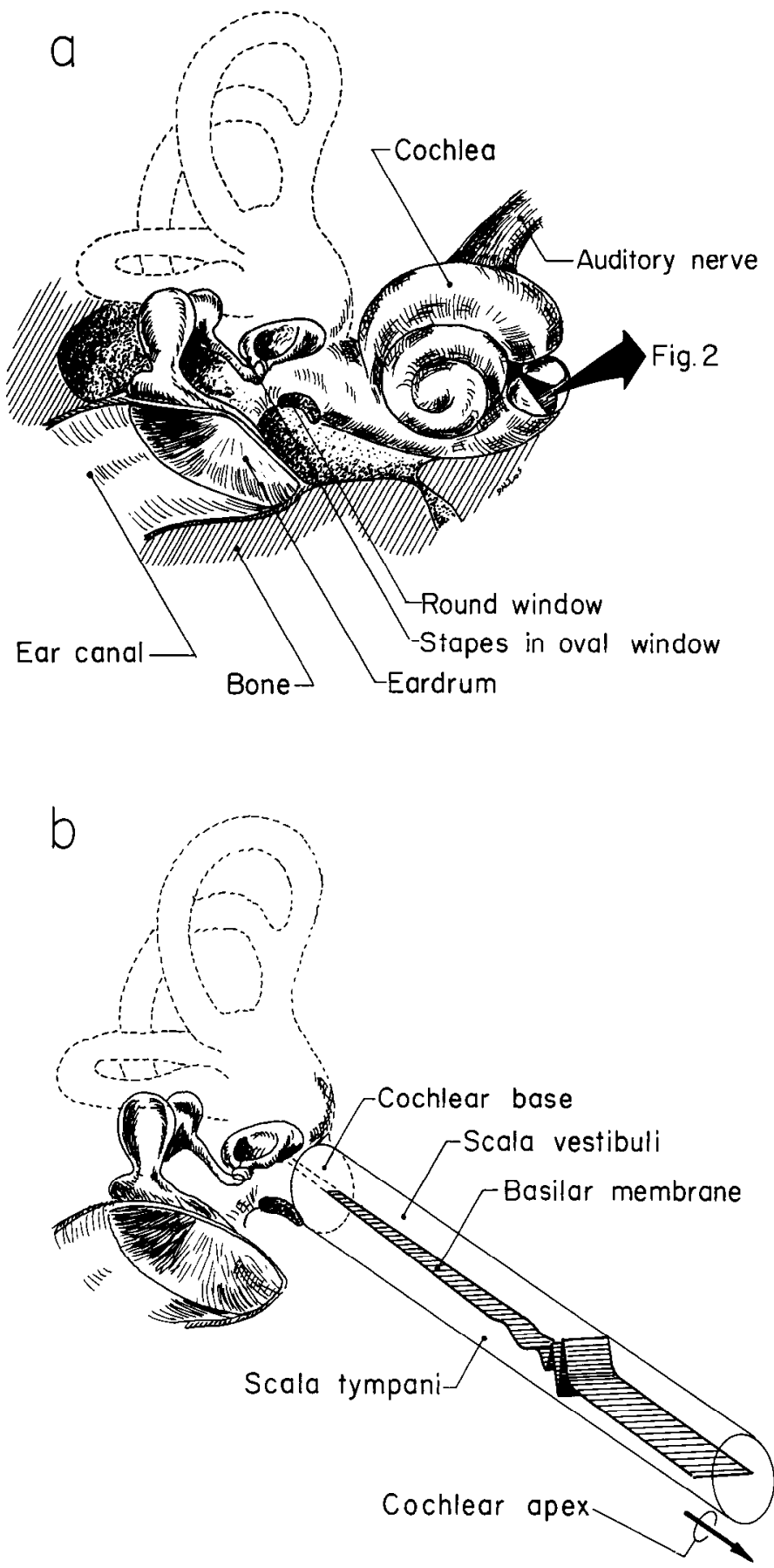

Figure 1. $a$, Outline of the peripheral auditory (solid lines) and vestibular (broken lines) systems. Sound is conducted to the cone-shaped eardrum that sets the three ossicles of the middle ear into vibratory motion. The innermost ossicle, the stapes, delivers these vibrations to the fluid-filled inner ear. The round window provides acoustic pressurc release. Details of the cochlear partition, containing the sensory cells within the organ of Corti, shown with hatching at the cut cross section of the cochlea, are given in Figure 2. $b$, The snail-shaped cochlea shown in $a$ is straightened out, and one of its internal dividing boundaries, the basilar membrane (see Fig. 2), is shown as a tapered ribbon. An instantaneous picture of the traveling wave is indicated by the shape of the basilar membrane, assuming that the sound input is a pure tone. The amplitude of the traveling wave is grossly exaggerated. extremes: they have finite duration and finite spectral breadth. However, there is a reciprocal relation in this continuum: the shorter the signal, the broader its frequency band, and conversely, the longer the signal, the narrower its frequency band. A device constructed to analyze signals is constrained the same way: in order to assure good frequency resolution of the signal, it must be processed in narrow frequency bands and observed for long duration; in contrast, to resolve short temporal features in the signal, it must be processed by relatively broad-band analysis. These are conflicting requirements for which ears must find an optimal solution. They must also perform over exceedingly broad intensity and frequency ranges, and with high resolution. Let us examine the severity of the requirements.

Under laboratory conditions, young adult humans can discriminate between two sounds whose frequencies differ by only about $0.2-0.5 \%$. This indicates that the neural representation of such two sounds prepared by the cochlea is sufficiently different to enable the cerebral cortex to distinguish between them. Let us assume that only spectral clues (not related to the timing of neural spikes) are used for analysis. Then, resorting to a relatively simple model of frequency discrimination, one can estimate that filters used to perform such analysis would have to possess bandwidths of approximately $1.2 \%$ of the center frequency of the filter. A filter of this sharpness, when excited by a brief transient, would persist in its ringing oscillatory response for a significant time period, corresponding to a time constant equivalent to 26 cycles. Such long persistence of the response would preclude a useful temporal analysis of brief sounds, yet timing differences of 6-10 $\mu \mathrm{sec}$ between signals presented to the two ears can be detected by human subjects. Furthermore, at detection threshold, the ear responds to sound causing displacements in the cochlea that are subatomic in dimensions. From recent measurements, one can estimate these displacements to be $10^{-10}$ to $10^{-11} \mathrm{~m}$ (Sellick et al., 1982). Broad-band thermal noise motion of the ciliary (hair) bundles of sensory receptor cells is estimated to be at least $10^{-9} \mathrm{~m}$ (Bialek, 1987). After prefiltering by the cochlea's mechanical frequency analysis, the thermal noise displacement of the ciliary bundle may be of the order of $10^{-10} \mathrm{~m}$. It follows that the sensory cells detect signals that may be smaller than, or of the same magnitude as, the background noise.

The ear is also capable of processing sounds over an at least a millionfold range in energy. Another requirement for optimal sound detection is the ability to respond over a relatively broad frequency range. While mammals process sounds that can encompass 10 octaves, nonmammalian vertebrates generally respond to much narrower frequency bands lying at the bottom end of the mammalian frequency response range. In fact, the desideratum of extending the frequency range may have been the primary pressure for developing mammalian hearing specializations.

\section{Anatomy}

\section{Cochlear duct and organ of Corti}

Figure $1 a$ shows a simplified sketch of the peripheral auditory system. Airborne sound entering via the ear canal propagates to the lateral boundary of the middle ear, the eardrum, which is set in vibratory motion. These vibrations are mechanically transmitted by the ossicles to the fluid-filled cochlea. In most mammals, the ossicles are a chain of three linked bones that are suspended by ligaments within the air-filled middle ear cavity. The inner ear, comprised of a vestibular (shown in broken 


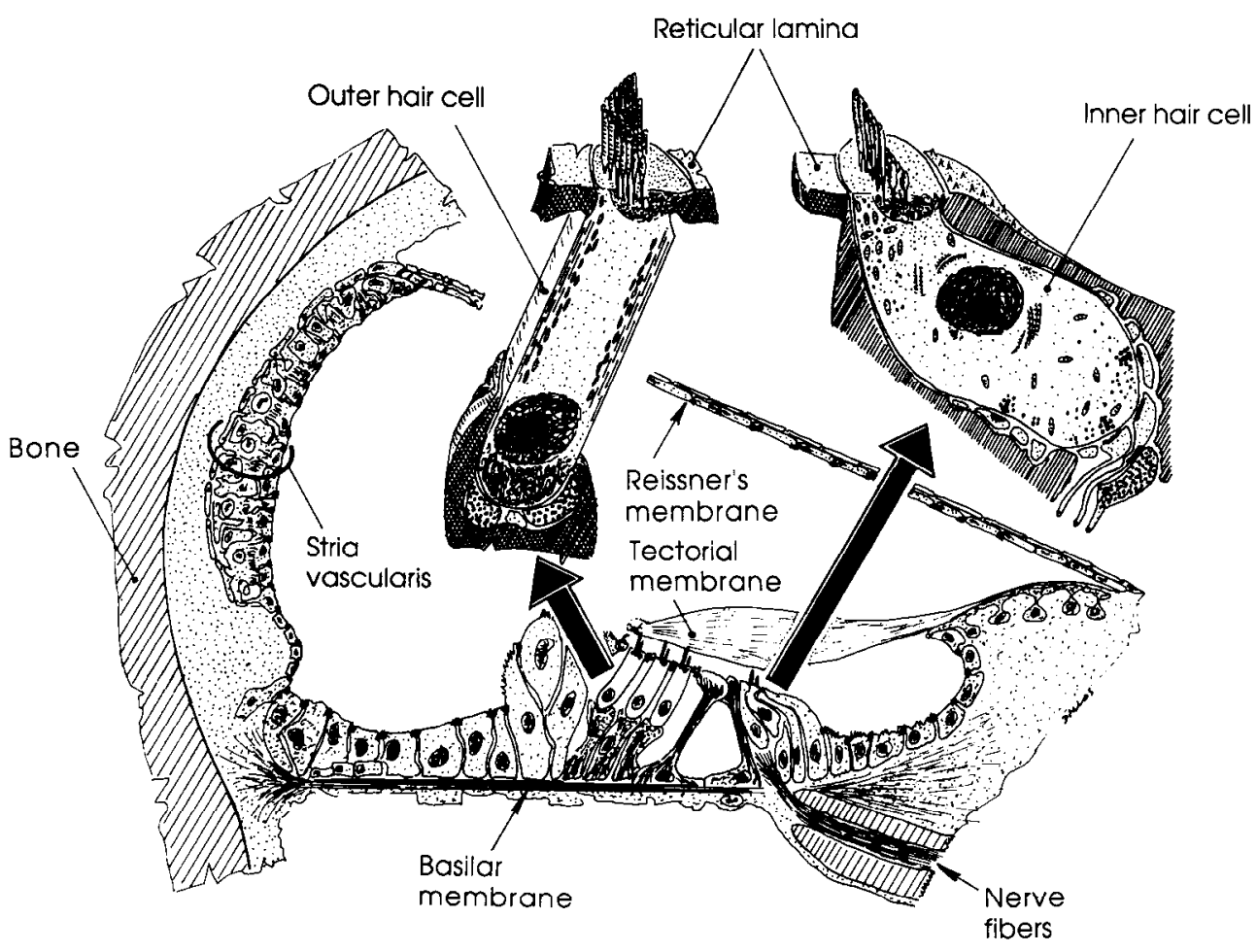

Figure 2. Cross section of the cochlear partition, or scala media, with one of three OHCs (left) and an IHC (right) shown enlarged. Boundaries of the partition are Reissner's membrane, basilar membrane, and the peripheral wall, lined by the stria vascularis. The organ of Corti is on the scala media side of the basilar membrane; it contains an array of supporting cells and the $\mathrm{OHCs}$ and IHCs The tectorial membrane is above the organ of Corti. Nerve fibers enter the organ from the central bony core of the cochlea. Inside the partition is endolymph; outside it and within the organ of Corti spaces is perilymph. The reticular lamina, formed by support cell processes, is a flat plate covering the organ of Corti. Modified from Dallos (1985). outline) and a cochlear portion, is a system of cavities in the temporal bone of the skull. The entire cavity is fluid filled. The middle ear and the inner ear communicate via two openings in the temporal bone, the oval and round windows. The innermost ossicle, the stapes, is inserted into the oval window and sealed by an elastic ligament. The round window is covered by a flexible membrane. The length of the cochlea itself is about $35 \mathrm{~mm}$ in humans; it coils as a snail shell about a center core through which the auditory portion of the eighth cranial nerve enters.

Key elements for understanding the neurobiology of the cochlea are situated within the cochlear partition. This triangularshaped duct, shown in the cutaway of Figure $1 a$ and in detail in Figure 2, is the cochlear portion of the membranous inner ear. The latter contains the sensory epithelia of the auditory and vestibular systems. The elaborate duct system roughly conforms to the shape of the bony inner ear. The space between the bony and membranous portions of the inner ear is filled by a filtrate of cerebrospinal fluid, the perilymph, while the membranous portion is filled by endolymph, the predominant cation of which is potassium $\left(\left[\mathrm{K}^{+}\right] \sim 160 \mathrm{mM} ;\left[\mathrm{Na}^{+}\right]<1 \mathrm{mM}\right.$; $\left.\left[\mathrm{Ca}^{2+}\right] \sim 20 \mu \mathrm{M}\right)$. The boundaries of the cochlear partition (also known as scala media) are the basilar membrane (separating it from one of the perilymph-filled channels the scala tympani), Reissner's membrane (separating it from the other perilymphatic channel the scala vestibuli), and the lateral wall of the cochlea. The auditory sensory epithelium, the organ of Corti, is a cellular matrix located on the scala media side of the basilar membranc. As shown in Figure 2, a complement of support cells and two types of sensory (hair) cells form a unit segment, which is repeated about 3500 times along the length of the organ of Corti. The scala media is separated from the surrounding structures by a luminal layer of cells that are sealed by tight junctions. This arrangement affords the biochemical isolation of the scala media from its surround. The stria vascularis, lining the lateral wall, is a threecell-layer, ion-transporting epithelium incorporating a vascular bed; it produces the endolymph, and as a byproduct of its transport process, it positively polarizes the endolymphatic fluid. This endolymphatic potential ranges from +80 to $+100 \mathrm{mV}$ with respect to the perilymph.

Reissner's membrane is acoustically transparent and does not influence the cochlea's mechanical functions; its likely role is to separate endolymph from perilymph. From the vantage point of cochlear fluid mechanics, the scalae vestibuli and media may be thought of as a single compartment. In contrast, the basilar membrane, while permeable to ions and thus allowing spaces within the organ of Corti to be filled with perilymph, is one of the salient hydromechanical elements in the workings of the cochlea. Its mechanical properties control the passive, von Békésy-type traveling wave that is sustained by it upon sound stimulation. When flattened and straightened, as in Figure $1 b$, the basilar membrane appears wedge shaped, with its width gradually increasing from the cochlea's "input" end (its base) loward the far end (its apex). The change in width results in a $10^{4}$ - to $10^{5}$-fold reduction in the membrane's stiffness from base to apex (Steele and Taber, 1981). This stiffness is conferred by the membrane's dense strands of collagen fibers running perpendicular to the long dimension and making the membrane highly anisotropic (Voldrich, 1978).

The geometry of the organ of Corti and its deformational properties are determined by the arrangement and structure of specialized supporting cells that send actin- and tubulin-packed phalangeal processes toward the roof of the organ of Corti. The processes flatten into an intermeshed network, the reticular lamina, that is closed to the endolymphatic space by tight junctions. The apices of sensory cells are sealed by tight junctions into the reticular lamina. The remaining cochlear structure of great importance is the tectorial membrane. This is a collagenous, acellular gel paralleling the reticular lamina. The space between tectorial membrane and reticular lamina is apparently open to endolymph. Consequently, the apical faces of hair cells and the 
entire reticular lamina are bathed in this fluid. The complex consisting of tectorial membrane, organ of Corti, and basilar membrane may be thought of as the accessory structures to the hair cells. Their role is to deliver a processed mechanical signal to the appropriate group of sensory receptor cells whose primary mechanical input is related to the relative motion between tectorial membrane and reticular lamina.

\section{Hair cells}

Hair cells are the common receptors of the auditory and vestibular sensory organs and of the lateral line. They are epithelial cells of somewhat variable morphology. Only the two types of mammalian cochlear hair cells, inner and outer hair cells (IHCs, $\mathrm{OHCs}$ ), are considered here (Fig. 2). In the human cochlea there are approximately $12,000 \mathrm{OHCs}$ and 3500 IHCs. A recent review by Hudspeth (1989) provides additional information about hair cells and their functioning. The flat, apical surfaces of both types of hair cell are crowned by a ciliary, or sensory hair, bundle. Each bundle contains cilia in an array that has a distinct organization and an axis of symmetry. This axis is largely radial in the coiled cochlea. IHC cilia form a shallow U-shape, and $\mathrm{OHC}$ cilia present a $\mathrm{W}$-, $\mathrm{V}$-, or $\mathrm{U}$-shape depending on their location. The foot of the configuration points toward the periphery of the cochlear spiral. Cilia are evaginations of the cell, sheathed by the plasma membrane. Their internal core consists of extensively cross-linked actin filaments (Flock and Cheung, 1977; Tilney et al., 1980), rendering the cilia rigid; upon deflection at their tip, they pivot at the tapered insertion points but do not bow (Flock et al., 1977). When displaced at the tallest cilia, the entire bundle moves as a unit, due to adjacent cilia being connected to one another with filamentous material (Pickles et al., 1984).

While the apical aspect of the hair cell is dominated by the cilia, its basal, infranuclear end has the hallmarks of presynaptic regions in the $\mathrm{IHC}$ and both pre- and postsynaptic regions in the $\mathrm{OHC}$. The extended supranuclear segment of OHCs is remarkable for its minimal cytoplasmic content and for its elaborate subplasma membrane specializations, which are thought to participate in the cell's motile activity. The illustration in Figure 3, along with the inset in Figure 2, should aid in visualizing this region. The most conspicuous feature is several layers of subsurface, or lateral, cisternae, having the appearance of smooth endoplasmic reticulum, that run concentrically along the cell membrane from the cuticular plate down to the synaptic region. One finds large numbers of mitochondria closely apposed to the innermost cistern. There is a gap between the outermost cistern and the plasma membrane, which houses an elaborate cortical network. This consists of a helical filamentous backbone, probably actin, having a pitch of about $75^{\circ}$ with the long axis. Adjacent turns are cross-linked with approximately 3-nm-diameter fibrils, conceivably spectrin or $\alpha$-actinin (Holley and Ashmore, 1988). The entire structure is linked to both the plasma membrane and the outermost cisternal wall by periodic pillars (Flock et al., 1986) 4-6 $\mathrm{nm}$ in diameter. The OHC latcral membrane has a high protein content, accounted for by approximately 6000 large protein particles per $\mu \mathrm{m}^{2}$, covering up to $75 \%$ of the membrane (Forge, 1991). The particles seem to be apposed to the pillars but do not penetrate the membrane (Arima et al., 1991). Since the estimated ion channel density in the lateral membrane is much less than that of these particles, it is likely that their majority subserves another function (conceivably motility). The lateral walls of IHCs possess clusters of intramembranous particles, possibly associated with ion channels. IHCs are surrounded by supporting cells, whereas OHCs are free along virtually their entire longitudinal extent; they are bathed in the perilymph that fills the cavities of the organ of Corti.

\section{Innervation}

The discovery of the disparity in innervation patterns between IHCs and OHCs (Spoendlin, 1970) clearly paved the way to our present concept of cochlear function, in which IHCs take the role of the sensory receptor in the hearing organ and in which the search is ongoing for understanding the primary function of OHCs (Dallos, 1985; Kim, 1986).

Neurons terminating on IHCs comprise $90-95 \%$ of the afferent pool. While about 20 afferents yield punctate, solitary bouton endings on each IHC, OHC afferents branch profusely and may innervate about $10 \mathrm{OHCs}$ in the cochlear base and as many as $\mathbf{5 0}$ in the apex. In the adult ear, no fiber innervates both IHCs and $\mathrm{OHCs}$. Central axons of both types of ganglion cells synapse in the ipsilateral cochlear nucleus of the medulla. An overview of afferent innervation is found in Spoendlin (1970). Efferent connections also differ between the two receptor cell types. The final descending path emerges in the superior olivary complex. Unmyelinated fibers originating in small cells near the lateral superior olivary nucleus descend, mostly ipsilaterally, toward IHCs. They terminate on the afferent dendrites coming from IHCs, rarely on the cell bodies themselves. Myelinated fibers stemming from larger cell bodies near the medial superior olive travel mostly contralaterally toward OHCs. These fibers terminate in large granulated endings that dominate the neural pole of OHCs. The efferent influence on OHCs is presynaptic (possibly affecting motility), while that on IHCs is postsynaptic. An overview of efferent innervation is found in Warr and Guinan (1979).

\section{Transduction in hair cells}

\section{Input to hair cells}

The excitatory proximal stimulus to any hair cell is the bending of the ciliary tuft toward the kinocilium (Hudspeth and Corey, 1977) or, in case of mammalian hair cells, toward the tallest row of stereocilia (Russell and Richardson, 1987). Forces exerted on the ciliary bundle derive from relative displacements between the reticular lamina and the tectorial membrane, either due to the direct attachment of cilia or, alternatively, due to tectorial membrane-driven flow of endolymph around the cilia. The latter forces are more efficient in stimulating cilia that are not attached to the tectorial membrane. In OHCs, the tallest row of cilia is firmly connected to the underside of the tectorial membrane, but it is possible that IHC cilia are either entirely freestanding or only tenuously attached (Lim, 1980). OHCs, due to their direct tectorial connection, can efficiently deliver forces via their cilia; the $W$-pattern, and the resulting stiffening of their ciliary bundle, may serve this function. OHC motile response presumably alters the input to IHCs by modifying the tectorial membrane-reticular lamina relationship (Zwislocki, 1990). Consequently, the strong coupling between $\mathrm{OHC}$ cilia and tectorial membrane and the concomitant decoupling of IHC cilia may be one corollary of the development of $\mathrm{OHC}$ mechanical feedback in the mammal. IHC cilia, with their flattened U-pattern, appear ideally sculpted to be stimulated by endolymph flow.

As a result of the viscoelastic coupling of forces to IHC cilia, 


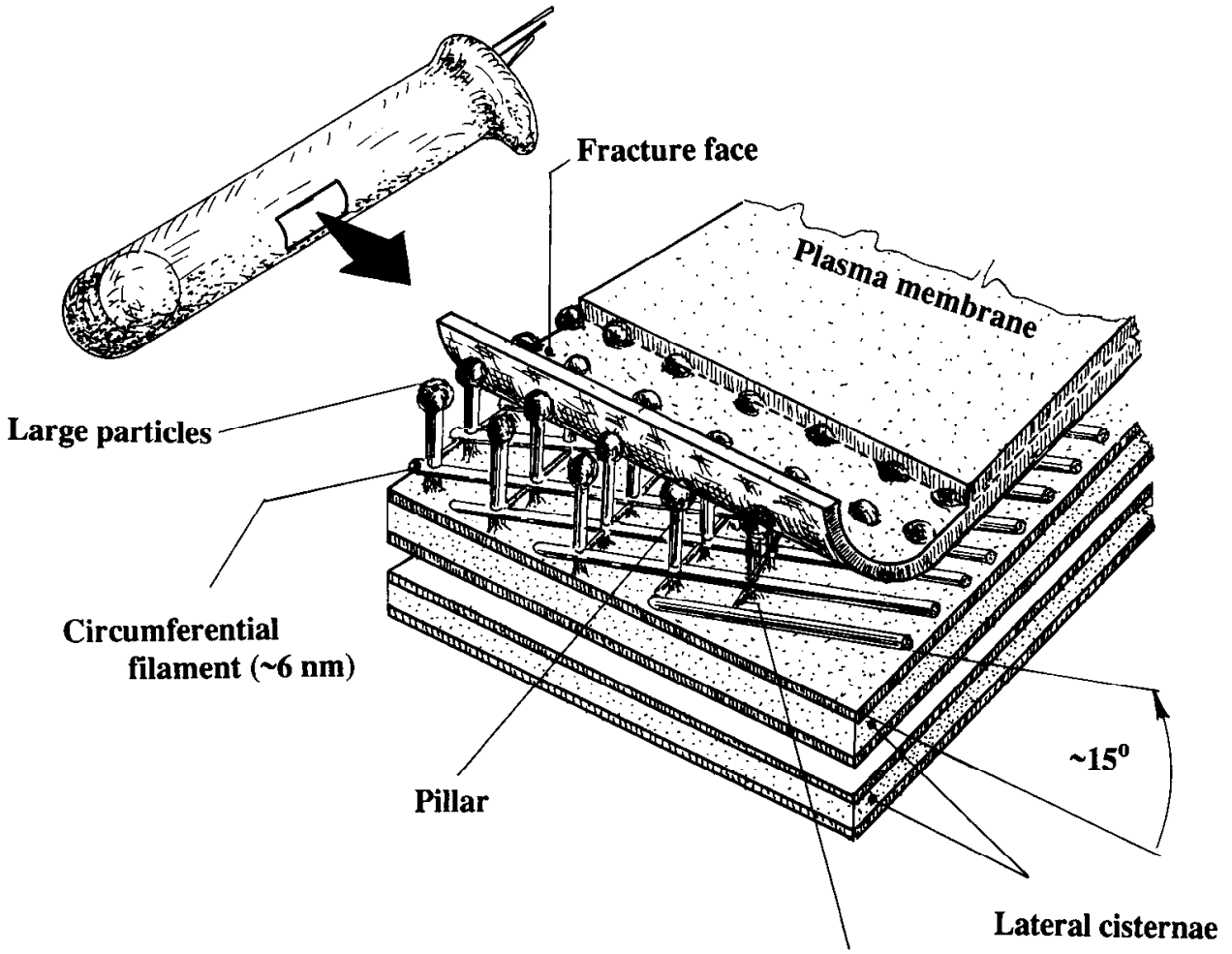

Cross link $(\sim 3 \mathrm{~nm})$
Figure 3. Subplasma membrane cortical structures of the OHC. The pitch of the thick helical circumferential filaments is approximately $15^{\circ}$. These filaments (probably actin) are cross-linked by thinner strands $(\alpha$-actinin or spectrin) and attached by pillars to the outermost lateral cistern and to large protein particles (candidate motor molecules) in the inner leaflet of the plasma membrane. The lateral cisternae are membrane-bound organelles that form multiple concentric layers within the cell's perimeter. Modified from Holley (1991) and Arima et al. (1991). at low frequencies their displacement is likely to be proportional to the velocity of motion of the basilar membrane. Because of this velocity dependence of IHC input, very low-frequency (DC) signals are probably ineffective in stimulating IHCs. In other words, DC displacements of basilar and/or tectorial membranes, which may arise from asymmetrical nonlinearities of the cochlear mechanics, are not likely to produce an IHC response. This means that the $\mathrm{DC}$ receptor potential, all important at high acoustic frequencies in producing an output (neurotransmitter release) from the IHC, is due to the IHC's own rectifier characteristics.

\section{Transduction}

A coherent and well supported hypothesis of hair cell transduction implicates mechanically gated channels located at or near the tips of stereocilia that are controlled by changing tension in attached elastic elements, termed "gating springs" (Hudspeth, 1989). Slender filamentous tip links of unknown structure (elastin?) reach up from the top of short cilia to the side of adjacent tall cilia, paralleling the mirror-symmetry axis (Pickles et al., 1984). The arrangement of these tip links, assumed to be the gating springs, is consistent with the cell's directional sensitivity: bundle displacement toward the tallest cilia, resulting in the stretching of the links, is excitatory; movement in the opposite direction is inhibitory, while orthogonal displacement is ineffective. Due to its direct activation by the mechanical stimulus, the opening and closing times of the gate are very short, certainly less than $50 \mu \mathrm{sec}$ (Hudspeth, 1989).

In all inner ears, the cilia, and hence the transducer channels, are in contact with endolymph, making potassium the likely carrier of transducer current (Fig. 4). The driving force for $\mathrm{K}^{+}$ is not an electrochemical potential but the electrical gradient across the ciliary membrane, produced by the sum of the cell's resting potential and the positive endocochlear potential, a total of 120-160 mV. Potassium loading of the cells from the endolymph is also useful in reducing their reliance on indigenous Na-K ATPase (Ashmore and Meech, 1986) and transferring the source of metabolic energy to the remote stria vascularis. The potential drop on the basolateral cell membrane, produced by the receptor current, may be shaped and altered by the passive reactance of the cell membrane and by voltage- and time-dependent conductances. One form of influence is due to the inevitable low-pass filtering, owing to the parallel resistance capacitance $(\mathrm{RC})$ of the basolateral cell membrane. The filter shunts those frequencies that are above its corner frequency: $f_{o}=$ $1 / 2 \pi \mathrm{RC}$, and consequently limits the size of the $\mathrm{AC}$ receptor potential at high frequencies. Thus, limitations on neural phaselocking are largely a consequence of this presynaptic filtcring action by IHC membrane (Palmer and Russell, 1986). As discussed below, filtering by OHC membrane influences high-frequency motility of this cell.

Two types of $\mathrm{K}^{+}$flux dominate $\mathrm{OHC}$ basolateral membrane currents; one is activated at membrane potentials between -90 and $-50 \mathrm{mV}$, and the other at membrane potentials more positive than $-35 \mathrm{mV}$ (Ashmore and Meech, 1986; Housley and Ashmore, 1992). Input conductances of OHCs change monotonically with cell position along the cochlea, with highest values $(\sim 50 \mathrm{nS})$ found in the base and lowest $(\sim 4 \mathrm{nS})$ in the apex. This spatial conductance gradient affects the receptor potentials in a frequency-specific manner (Housley and Ashmore, 1992). Basolateral membrane conductances in IHCs (Kros and Crawford, 1990) are dominated by two different voltage-gated $\mathrm{K}^{+}$ channels, active in the -60 to $-20 \mathrm{mV}$ membrane potential range that encompasses in vivo values. Diagrams of IHC and $\mathrm{OHC}$, shown in Figure 4, depict the various currents and channels that dominate hair cell electrical phenomena and also sym- 
Figure 4. OHC and IHC cellular machinery. Solid arrows indicate the excitatory direction of ciliary bundle deflection. Hatched arrows symbolize $\mathrm{K}^{+}$ flow from endolymph into the hair cells. There is a resting $\mathrm{K}^{+}$flux down the electrical gradient, and this is modulated by the transducer channels that are directly gated by cilia deflection. OHCs and IHCs have different resting potentials. OHC basolateral membranes contain voltage-gated $\mathrm{Ca}^{2+}$ channels and Ca-activated $\mathrm{K}^{+}$channels. Depolarization $(\delta V)$ due to the receptor current ( $\mathrm{K}^{+}$flux) and subsequent calcium and potassium fluxes produces the ultimate receptor potential. In OHCs, the receptor potential activates the motile mechanism ( $V-M$ converter) that produces cellular shape changes and delivers mechanical feedback. The dominant innervation of OHC is efferent. IHC basolateral membranes contain at least two types of voltage-gated $\mathrm{K}^{+}$channels and $\mathrm{Ca}^{2+}$ channels in the synaptic region. IHCs are almost exclusively innervated by afferents.

\section{ENDOLYMPH \\ $+80 \mathrm{mV}$}

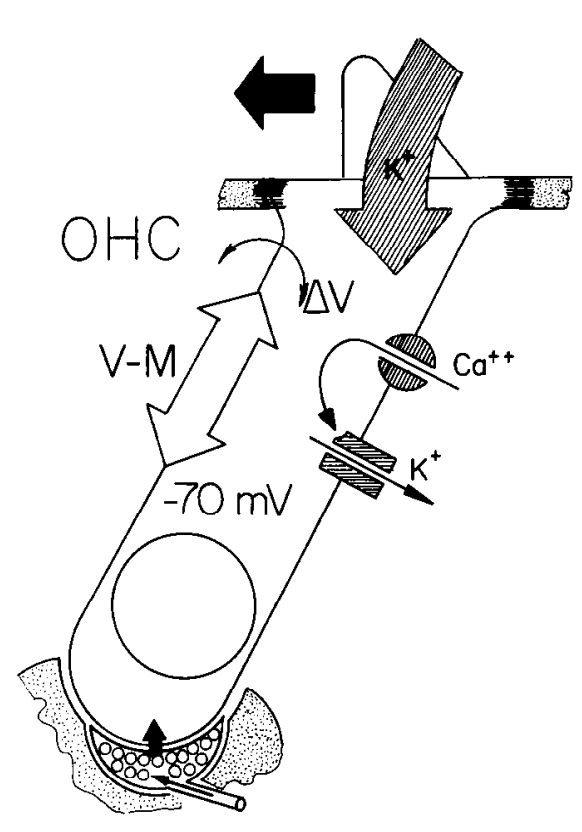

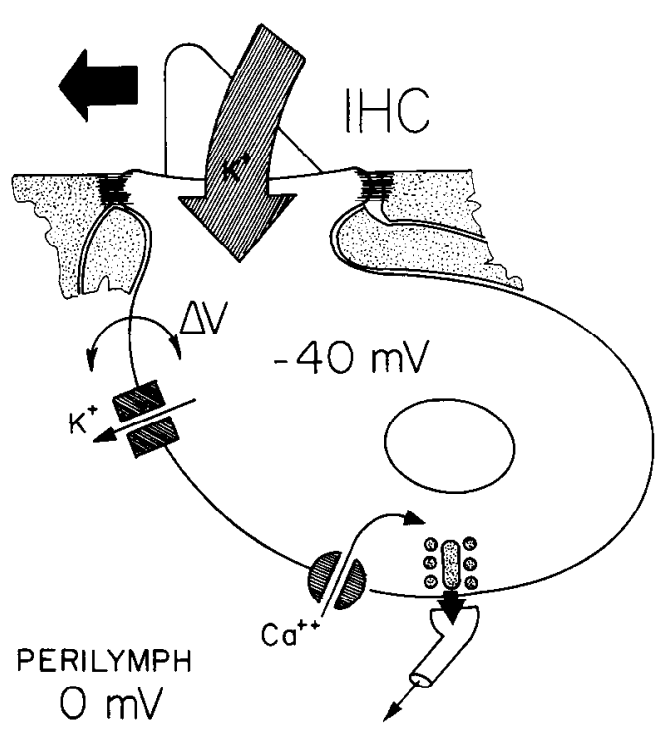

bolize the mechanism of the voltage-to-movement transducer associated with the $\mathrm{OHC}$ cell membrane, which is assumed to be the substrate of the cell's mechanical feedback function.

\section{Cochlear mechanics}

\section{Passive mechanics}

The contemporary view of the operation of the mammalian ear is that a hydromechanical event, von Békésy's traveling wave, devolving from the physical structure of the cochlea, provides the basis for frequency analysis. This rather crude mode of analysis appears to be augmented by a local cochlear feedback process that relies on OHCs as the effector elements. Deformations of the organ of Corti, caused by the traveling wave augmented by $\mathrm{OHC}$ feedback, produce the relative motions between tectorial membrane and reticular lamina that effect mechanoelectric transduction in both types of hair cell. In this view, IHCs transduce the preanalyzed signals and, via their dominant afferent connections, convey acoustic information to the CNS. Our first task is to understand the physical genesis of the "passive" von Békésy wave, before discussing the "active" feedback process thought to be mediated by OHCs.

Sound-induced, pistonlike motion of the stapes produces pressure changes in the adjacent cochlear fluid. The resulting acoustic events are dominated by a wave that develops as a pressure gradient across the basilar membrane-tectorial membrane sandwich, functioning as an "acoustic partition" between scala tympani and scala media. This gradient is made possible by the presence of the flexible round window membrane, which fronts on the air-filled middle ear space, and is at approximately atmospheric pressure. At the far, apical end of the cochlea, the endolymphatic space ends blindly and there is communication between the two perilymphatic scalae through an opening that provides an acoustic shunt across the cochlear partition for infrasonic frequencies. For that reason, extremely slow variations in sound pressure are ineffective in displacing the acoustic partition.

The pressure gradient across the acoustic partition produces a force upon it, setting it in motion and originating a wave that propagates away from the window region (Fig. $1 b$ ). The wave's speed of travel and properties are determined by the physical characteristics of the flexible boundary. Since with increasing distance from the window region, the stiffness of the basilar membrane decreases, so does the speed of travel, $v$, of this wave whilc its amplitude increases. At a given frequency, $f$, as the wave's velocity decreases, so does its wavelength, $\lambda$, since $\lambda=$ $v / f$. Acoustic energy propagation progressively slows until the wave effectively halts with the energy "piling up" at a particular, "characteristic" place (Lighthill, 1991). As the energy is concentrated in a very narrow region, traveling at very low speed, any mechanical loss (damping) of the basilar membrane is sufficient to dissipate it since this process of dissipation can take a long time. At the location of the membrane's own resonant frequency, it is purely dissipative, and it is there where the wave is extinguished; that is, its amplitude falls to zero. Any location has a characteristic frequency (CF), largely determined by the membrane's local stiffness. The CFs are arranged according to a spatial map, so that they progressively decrease from cochlear base to apex, just as stiffness decreases along the length. Thus, any location, $x$, has a resonant frequency, which is the limiting frequency for waves propagating toward it. A wave whose frequency is the same as the resonance frequency will achieve zero wavelength at $x$, that is, it becomes extinguished. Waves with lower frequency propagate through this point; waves with higher frequency do not reach it.

These considerations indicate that a particular basilar membrane motion pattern may be expected on simple physical grounds. This traveling wave sustained on the basilar membrane was first measured by von Békésy (1960) in cadaver ears, using 
optical methods that necessitated very high input levels to make the vibrations large enough to be visible.

\section{Response pattern in the live cochlea}

von Békésy's measurement of the traveling wave indicated linearity. In other words, except for amplitude scaling, the traveling wave was not dependent on stimulus level. It also showed shallow tuning. It is now known that he described the behavior of the "passive," or dead cochlea. In the living ear the wave motion of the basilar membrane is different both qualitatively and quantitatively from that seen in the dead ear. It shows an exceedingly nonlinear dependence on stimulus level and is much more sharply tuned (Sellick et al., 1982). Examples are shown in the top panel of Figure 5, which depicts basilar membrane amplitude at one particular location along the cochlea at different sound frequencies and levels. The plots are normalized to input stimulus level and, thus, approximate gain functions between basilar membrane displacement and stimulus. The gain is conspicuously dependent on stimulus level. If measured at a high level, the displacement is similar to that found by von Békésy. As stimulus level decreases, however, the gain functions become increasingly sharper. The frequency at the peak of the sharpest (lowest level) gain curve defines the CF of the basilar membrane location. It is noted that the gain increases in the vicinity of the CF only, and that for frequencies less than an octave below CF the gain is independent of level. Hence, the response reflects a band-limited nonlinearity around the CF (Rhode, 1971).

This nonlincarity, as well as the sharpness of tuning, depends on the physiological condition of the cochlea (Rhode, 1973). This is demonstrated in the bottom panel of Figure 5. Two sets of gain curves are shown, 20 decibels apart in stimulus level, for live and for dead ears. As in the top panel of Figure 5, in the live cochlea the gain is higher at the lower stimulus level. Upon death, however, this gain difference disappears and the tuning becomes independent of level. Putting it another way, the nonlinearity disappears with death.

To summarize, at any point along the basilar membrane, one specific frequency produces maximal response. The frequency selectivity of this response, reflected by the sharpness of the gain curve, is greatest near stimulus threshold and gradually decreases with larger inputs. The nonlinearity, expressed in the leveldependent bandwidth of the gain curves, is dependent upon the state of the cochlea. In a dead ear, all gain curves are nested and they are all equally poorly tuned. The frequency-selective gain increase (amplification) of the live cochlea over the dead one is now assumed to be due to mechanical feedback by OHCs.

\section{Active mechanics and $O H C S$}

The bases of our present concept of an active cochlear function were developed in the 1970 s and early 1980 s. Only a few salient ideas are presented here; a detailed review is found in Dallos (1988). Two major notions comprise the basis of the present framework. First, normal cochlear function is dependent on an active, mechanical feedback process, and second, OHCs are the agents of feedback. Let us examine the evidence for these two concepts.

The physiological vulnerability of gain, tuning, and nonlinearity has been discussed above. This vulnerability indicates that the hydromechanical processes of the cochlea are dependent on normal metabolism and are exquisitely sensitive to external interference of virtually any type. Passive systems do not have such characteristics. The mechanical nature of the active coch-
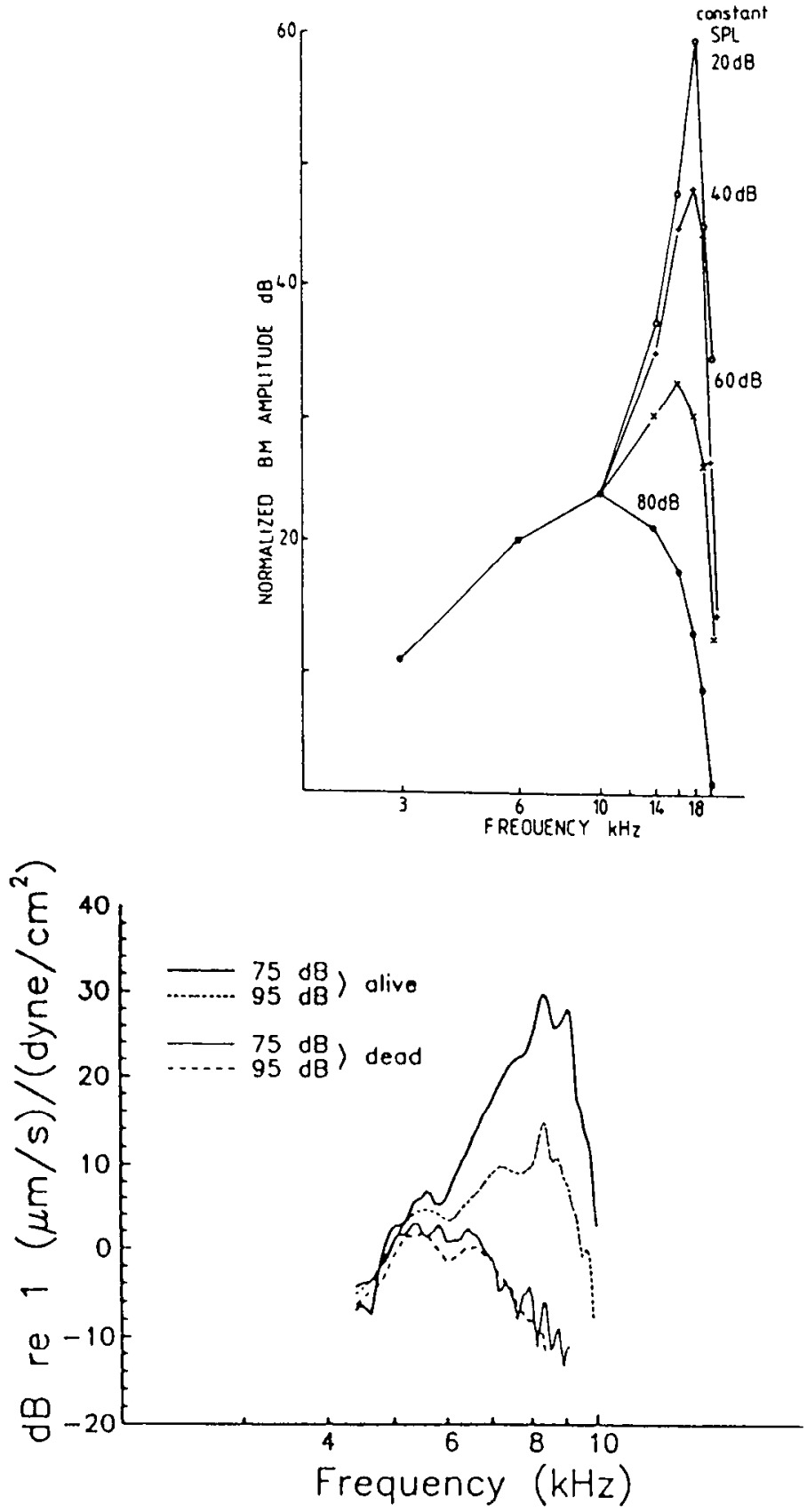

Figure 5. Top, Normalized basilar membrane amplitude at the sound pressure levels indicated as parameters. All curves converge below 10 $\mathbf{k H z}$, indicating linear response and equal gain, irrespective of level. Measurements were performed using the Mössbauer technique in the basal turn of the guinea pig cochlea. Maximal response frequency, $\sim 17$ kHz. From Johnstone et al. (1986). Bottom, Gain functions of basilar membrane displacement measured in the basal turn of the chinchilla cochlea with laser Doppler velocimetry. Maximal response frequency, $\sim 8.5 \mathrm{kHz}$. Measurements are shown at two sound levels, 75 and 95 $\mathrm{dB}$, and in conditions of live and dead cochlea. From Ruggero and Rich (1991).

lear process was indicated by the detection of sound in the ear canal, due to spontaneous oscillations, apparently of cochlear origin, retransmitted by the middle ear. These so-called spontaneous otacoustic emissions (Wilson, 1980) are the strongest evidence available that vibrations may be produced in the cochlea. Sound emissions from the ear that originate in the cochlea in response to acoustic inputs also support the validity of active 
proccsses providing local amplification (Kcmp, 1978). Finally, experiments showing that electrical stimulation of the cochlea with AC potentials can result in acoustic emissions and that these are physiologically vulnerable (Hubbard and Mountain, 1983) relate the active mechanical process to intracochlear electrical signals as likely precursors.

The intimate involvement of $\mathrm{OHCs}$ in the modification of cochlear output was suggested by observations that, in spite of IHCs providing 90-95\% of afferent sensory innervation, elimination of OHCs results in significant hearing loss (Ryan and Dallos, 1975) and alteration of tuning (Dallos and Harris, 1978; Harrison and Evans, 1979). Even though efferents almost exclusively terminate on $\mathrm{OHCs}$, their stimulation results in detuning of the IHC response (Brown et al., 1983) and altering the production of otacoustic emissions (Mountain, 1980). These data suggest that interference with $\mathrm{OHC}$ function profoundly affects the active mechanical process. Evidence for the identity of OHCs as the mechanical effectors in the active process became compelling with the discovery of in vitro alterations of their soma shape, induced by membrane potential changes, and by their chemical manipulations (Brownell et al., 1985; Zenner et al., 1985).

An active mechanical process that may be electrically elicited and that is entirely dependent on functioning OHCs is most parsimoniously identified with the OHC itself. These cells are then conceived to perform two transducer functions. First, they are mechanoelectrical transducers, as are all hair cells; they transduce mechanical input, the ciliary deflection, into an electrical signal, the receptor potential. Second, they are electromechanical transducers in producing a mechanical output, presumably somatic shape change, in response to their own receptor potential. This dual sensor-effector operation of the $\mathrm{OHC}$ is the apparent basis of the amplification of the passive traveling wave by the postulated active feedback.

\section{Modeling of the live cochlea}

Theoretical considerations indicate that the frequency response patterns, shown in Figure 5 under conditions that yield optimal tuning, are so shaped that they cannot be accounted for by models in which all basilar membrane vibratory energy is derived from the original sound input (de Boer, 1983; Neely and Kim, 1983). This includes passive linear and nonlinear models that allow for more than one degree of freedom in describing basilar membrane-related movements and tectorial membrane resonance (Allen, 1980; Zwislocki, 1980).

Instead, the performance of the living ear can be accounted for by active models that assume a local supply of energy that may be utilized to boost basilar membrane motion selectively. It is generally believed that energy needs to be supplied to the traveling wave in a region that is basal to the CF. Such active models usually incorporate the formalism of providing "negative damping," referring to some means of counteracting the inherent dissipation of energy in the basilar membrane and cochlear fluids (de Boer, 1983; Neely and Kim, 1983). The basic idea is to provide a cycle-by-cycle force to the basilar membrane in opposite phase with the resistive force produced by the passive damping; the two forces thereby cancel, and much larger response amplitude is achieved. Alternatively, it is possible to obtain the desired response character by assuming that the cochlear amplifier feeds back a reactive force to the basilar membrane. Such a force would reduce the impedance of the basilar membrane, at a location basal to the CF, and thereby boost the response amplitude (Kolston et al., 1990).

The feedback is needed at and just above threshold in order to improve the signal-to-noise ratio of detection, and is gradually disabled as stimulus level increases. Disabling is achieved by the saturation of the feedback element at high stimulus levels (Zwicker, 1979). The gain of the feedback path becomes zero and hence the feedback loop is opened. Saturation is also essential in stabilizing the feedback loop.

\section{Active mechanics and micromechanics}

\section{OHC motility}

The demonstration that $\mathrm{OHCs}$ isolated from the cochlea are capable of shape changes at audio frequency rates upon electrical stimulation was a key discovery (Kachar et a1., 1986; Zenner, 1986; Ashmore, 1987). These electromotile responses consist of primarily length changes, up to $3-5 \%$ of the total cell length under maximal electrical stimulation. The controlling variable is transmembrane potential change (Santos-Sacchi and Dilger, 1988). Depolarization of the cell results in contraction; hyperpolarization results in elongation, while small diameter changes are also measurable (Ashmore, 1987). The motile response is nonlinear, showing rectification in the contraction direction and also exhibiting saturation (Evans et al., 1989; Santos-Sacchi, 1989). The sensitivity of the response is approximately $2-20$ $\mathrm{nm} / \mathrm{mV}$ at low stimulus frequencies, but the process may be low pass, in that at constant-amplitude electrical potential input, the response decreases as stimulus frequency increases (Ashmore, 1987). While membrane potential is stepped to different levels under voltage clamp, the correlated behavior of motile response and nonlinear capacitive ("gating") currents suggests that motility involves the movement of membrane-bound charged molecules (Santos-Sacchi, 1991). Other work also suggests that $\mathrm{OHC}$ electromotility is produced by the concerted direct action of a large number of independent molecular motors that are closely associated with the cell's basolateral membrane (Holley and Ashmore, 1988; Dallos et al., 1991), conceivably with the densely packed particles seen therein. Displacements produced by the motor molecules may be coupled to the related cell cortex (Fig. 3). The response is not dependent on ATP or $\mathrm{Ca}^{2+}$, and is of speed so high as to rule out all hitherto described motile mechanisms (Kachar et al., 1986; Holley and Ashmore, 1988).

When $\mathrm{OHCs}$ are incorporated in their natural environment, their shape changes alter micromechanical processes: their displacement affects the traveling wave-induced deformation of the organ of Corti, feeding back energy so that the resulting compound deformation, and indeed the resulting aggregate traveling wave, is amplified. The details of this micromechanical process are poorly understood, but its end result, dictated by the hair cells' directional sensitivity, of producing highly localized radial sheer motion between reticular lamina and tectorial membrane, is clear. In vivo, the electromotile response is likely to be driven by the receptor potential produced in OHCs that results from acoustic stimulation. These responses consist of both cycle-by-cycle (AC) and tonic (DC) components. As symbolized in Figure $6 a$, length changes resulting from alternating depolarization and hyperpolarization may feed back an $\mathrm{AC}$ mechanical force upon the basilar membrane-tectorial membrane system. In addition, due to the rectifier properties of the transduction process and of electromotility, a DC length change would be produced as well (Fig. $6 b$ ). The AC force can counteract 
viscous damping while the DC may alter the basilar membranetectorial membrane relationship or the effective stiffness of the basilar membrane (to which OHCs are coupled) and, thus, set the operating point of the micromechanical system (Dallos, 1988). The DC receptor potential of $\mathrm{OHCs}$ is positive around the CF and negative at lower frequencies (Dallos et al., 1982); consequently, the operating point may be adjusted in opposing directions, enhancing or impeding the amplification process. $\mathrm{OHC}$ receptor potentials as well as the motile mechanism itself saturate at high levels, thus providing the necessary limiting of the feedback process. Aside from stimulus-evoked fast motile responses, a wide variety of agents are capable of stimulating slow contractions of OHCs (Brownell et al., 1985; Zenner et al., 1985; Flock et al., 1986). The response elicited by focal application of $\mathrm{ACh}$, the transmitter substance for cochlear efferents, is of particular interest. The possibility exists that the state of the organ of Corti may be mechanically modified by the CNS, via $\mathrm{DC}$ shape changes of $\mathrm{OHCs}$ mediated through efferent influence (Kim, 1986) as well as stimulus-evoked DC responses (Fig. 6c).

The receptor potential contains significantly more energy than the eliciting acoustic stimulus. This is due to the modulation of local metabolic energy by the hair cell transduction process. At least at low frequencies, the receptor potential is capable of supplying energy greatly in excess of that required to overcome the OHCs own internal stiffness in producing extension-elongation cycles that are commensurate with basilar membrane movements in vivo. How this is affected by the organ of Corti's mechanical load upon the cell in situ is unknown, however.

\section{Problems and uncertainties}

An often mentioned problem in associating $\mathrm{OHC}$ electromotile responses with a feedback role in vivo is the high-frequency attenuation of the receptor potential due to the cell's basolateral membrane capacitance and the possibly low-pass character of the electromotile process itself. The dual high-frequency attenuation clearly reduces the cell's ability to produce sufficient motile feedback at the high end of the audio range. For example, one can extrapolate to $20 \mathrm{kHz}$ from available data at $100 \mathrm{~Hz}$, that at the threshold of hearing the mechanically unloaded $\mathrm{OHC}$ produces about $0.0015 \mathrm{~nm}$ motion under the least favorable estimate and about $0.3 \mathrm{~nm}$ amplitude motion for the most favorable. The latter number is commensurate with the displacement of the basilar membrane at threshold $(\sim 0.2 \mathrm{~nm})$, but less optimistic estimates are not, calling into question the efficacy of the entire feedback scheme. The problem of adequate feedback amplitude at high frequencies is exacerbated by the need for the feedback force, if it is to counteract viscous damping, to increase in proportion to velocity, that is, stimulus frequency.

Admittedly, the in vitro experiments on OHCs may underestimate their gain and misestimate their frequency response. It is also possible that some other aspects of motility, hitherto unobserved in mammals, are the critical variables, for example, ciliary movement or stiffness change. Such phenomena have been detected in nonmammalian hair cells (Crawford and Fettiplace, 1985). It may be that the key effect is that the prominent DC component of OHC motility modifies the system in a parametric feedback arrangement (Fig. 6b,c; Dallos, 1988). One could conceive in a system of more than one degree of freedom that coupling of organ of Corti to tectorial membrane could be affected via the stimulus-controlled alteration of ciliary stiffness (Mountain et al., 1983). Alternatively, the effective mechanical impedance of the basilar membrane can be modified if the pri-

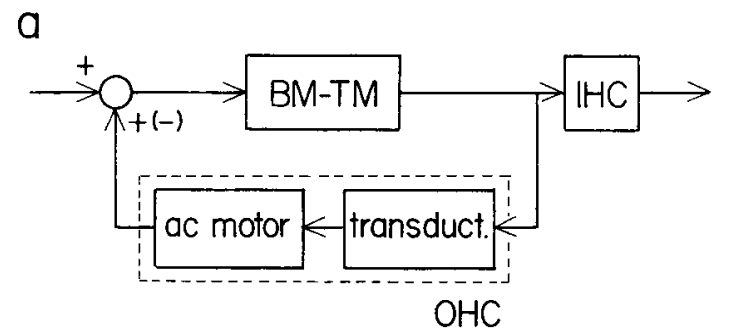

b
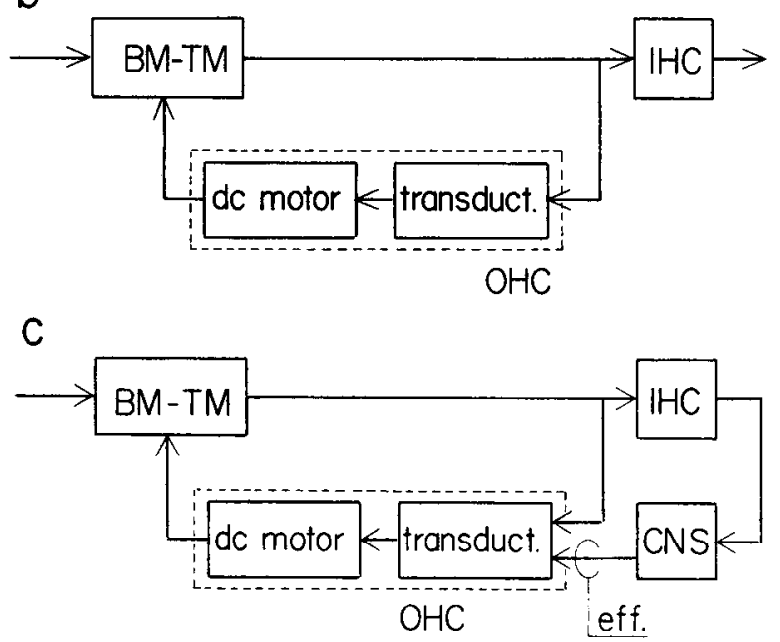

Figure 6. Block diagrams of the cochlear feedback system. $a$, The feedforward path is the basilar membrane-tectorial membrane system $(B M-T M)$, and the feedback path is the OHC. The OHC performs both sensor (transducer) and effector (motor) functions. The IHC is the detector of the output of the feedback system. In this panel, it is assumed that the motor $(\mathrm{OHC})$ produces a cycle-by-cycle $(\mathrm{AC})$ force that is summed with the basilar membrane displacement in some appropriate phase, producing either positive or negative feedback, depending on frequency. $b$, Same as in $a$, but here the assumption is that the OHC produces a DC feedback force that alters the operating point of the basilar membrane-tectorial membrane system. This is a parametric feedback arrangement. $c$, Same as in $a$, but the modifying effect of the efferent system upon OHCs is incorporated as a part of the total DC control system. From Dallos (1988).

mary effect of $\mathrm{OHC}$ motility in vivo is a stiffness change (Allen, 1990; Kolston et al., 1990).

In addition to the problem of feedback force amplitude at high frequencies, a conceivably even more formidable problem is the necessity of providing cycle-by-cycle OHC feedback with an appropriate phase, determined by stimulus frequency. This is equivalent to the necessity that the feedback be frequency specific and bear a deterministic relation to the basilar membrane's resonant frequency. OHC electromotility in vitro has not been shown to possess tuning. However, some data do indicate that isolated OHCs have tuned motile responses upon mechanical stimulation of their basolateral membranes but not of their ciliary bundles (Brundin et al., 1989). These responses, in contrast to electromotility, are reported to be sharply tuncd, intimating a band-pass process intrinsic to the cell itself. These findings are coherent with measurements in an intact but in vitro cochlea, in which primary tuning has been attributed to hair cells, as opposed to the traveling wave (Khanna et al., 1989). It still remains to demonstrate the existence of $\mathrm{OHC}$ motile responses to mechanical stimulation of their ciliary bundle and to examine the frequency-response characteristics of this "mechanomotility." At the time of this writing, the most unsettled 
and interesting aspects of the cochlear puzzle are the identity of the motor elements (molecules) themselves, the means of coupling of the force exerted by the motor molecules to the OHC cortex, and the mode and nature of interaction between OHC feedback and organ of Corti-basilar membrane micromechanics. The need for feedback is well demonstrated, and the OHC is almost certainly the mediator. The actual details of mediation, though, are still wanting.

\section{Conclusions}

Both theoretical and experimental results dictate a reassessment of the fundamental operation of the mammalian cochlea. The hydrodynamic frequency-to-place transformation performed by the von Békésy traveling wave is now seen as a preliminary step, a somewhat rudimentary preanalysis of the acoustic spectrum. This analysis is inherent in the physical nature of the cochlear configuration, and it is performed irrespective of sound level and the physiological state of the cochlea. The process is linear and it serves as a "first cut" in exciting sensory receptors in a frequency-specific manner. In order to obtain the degree of frequency selectivity and sensitivity over an extended high-frequency range that is required by mammals, the traveling wave is augmented by an active amplification process. Prevailing thought holds that the amplification is expressed in the provision of vibratory energy to the traveling wave at a location basal to its peak. Due to this, inherent viscous losses are compensated, and a much more sharply tuned and higher sensitivity vibratory pattern results.

The instrument of this amplification appears to be the OHC. These cells are shown to have the ability to undergo significant shape changes upon alterations of their membrane potential. Such elongation-contraction cycles occur in vitro at audio frequency rates, presenting at least a potential for cycle-by-cycle feedback of vibratory energy from $\mathrm{OHC}$ to basilar membrane. It should be emphasized, however, that the actual details of the amplification mechanism are not yet understood. The electromotile process is novel and different from other known cellular or molecular motors. Because of the asymmetry of the OHC's transducer and of its motor, the feedback contains a significant DC component that could be instrumental in setting the operating point of cochlear micromechanics. Due to the saturating nonlinear nature of both the OHC transducer and motor, the effective feedback action is limited to low input levels, roughly $40 \mathrm{~dB}$ above threshold.

In this scheme IHCs, innervated by $90-95 \%$ of cochlear afferents, are the true sensory receptors of the auditory system. They detect relative motions between tectorial membrane and organ of Corti that reflect micromechanical events. The latter are controlled by the OHCs, which amplify the traveling wave. OHCs may report their mechanical state, instead of acoustic information, to the CNS via their sparse afferent connections. If so, the changing number of OHCs per afferent fibers along the cochlea may serve to signal the condition of OHCs performing similar activity. The advantages of evolving this feedback process are high sensitivity, high-frequency capabilities, and optimized temporal processing. One cost is pronounced physiological vulnerability. The effector elements, OHCs, appear to be the most susceptible components of the ear to damage by environmental, disease, or aging processes. Another price is that the system is forced to operate in a nonlinear mode. This means that the mammalian CNS had to evolve mechanisms to cope with the introduction by the cochlea of frequency com- ponents that are not present in a multicomponent sound spectrum and with a severe distortion of the contour of the input spectrum due to mutual nonlinear interference among frequencies. Some such interference can result in acoustic contrast enhancement and thus be beneficial in the processing of complex sounds, such as speech.

\section{References}

Allen JB (1980) Cochlear micromechanics-a physical model of transduction. J Acoust Soc Am 68:1660-1670.

Allen JB (1990) Modeling the noise damaged cochlea. In: Mechanics and biophysics of hearing (Dallos P, Geisler CD, Matthews JW, Ruggero MA, Steele CR, eds), pp 324-331. New York: Springer.

Arima T, Kuraoka A, Toriya R, Shibata Y, Uemura T (1991) Quickfreeze, deep-etch visualization of the 'cytoskeletal spring' of cochlear outer hair cells. Cell Tissue Res 263:91-97.

Ashmore JF (1987) A fast motile event in outer hair cells isolated from the guinea pig cochlea. J Physiol (Lond) 388:323-347.

Ashmore JF, Meech RW (1986) Ionic basis of the resting potential in outer hair cells isolated from the guinea pig cochlea. Nature 322:368371.

Bialek W (1987) Physical limits to sensation and perception. Annu Rev Biophys Biochem 16:455-478.

Brown MC, Nuttall AF, Masta RI (1983) Intracellular recordings from cochlear inner hair cells: effects of stimulation of the crossed olivocochlear bundle. Science 222:69-72.

Brownell WE, Bader CR, Bertrand D, de Ribaupierre Y (1985) Evoked mechanical responses of isolated cochlear outer hair cells. Science 227:194-196.

Brundin L, Flock $\AA$, Canlon B (1989) Sound-induced motility of isolated cochlear outer hair cells is frequency-specific. Nature 342:814816.

Crawford AC, Fettiplace R (1985) The mechanical properties of ciliary bundles of turtle cochlear hair cells. J Physiol (Lond) 364:359-379.

Dallos P (1985) The role of outer hair cells in cochlear function. In: Contemporary sensory neurobiology (Correia MJ, Perachio AA, eds), pp 207-230. New York: Liss.

Dallos P (1988) Cochlear neurobiology: some key experiments and concepts of the past two decades. In: Functions of the auditory system (Edelman GM, Gall FW, Cowan WM, eds), pp 153-188. New York: Wiley.

Dallos P, Harris D (1978) Properties of auditory nerve responses in the absence of outer hair cells. J Neurophysiol 41:365-383.

Dallos P, Santos-Sacchi J, Flock $\AA$ (1982) Cochlear outer hair cells: intracellular recordings. Science 218:582-585.

Dallos P, Evans BN, Hallworth R (1991) Nature of the motor element in electrokinetic shape changes of cochlear outer hair cells. Nature 350:155-157.

Davis H (1983) An active process in cochlear mechanics. Hearing Res 9:79-90.

de Boer E (1983) No sharpening? A challenge to cochlear mechanics. J Acoust Soc Am 73:567-573.

Evans BN, Dallos P, Hallworth R (1989) Asymmetries in motile responses of outer hair cells in simulated in vivo conditions. In: Cochlear mechanisms (Wilson JP, Kemp DT, eds), pp 205-206. New York: Plenum.

Fettiplace $\mathbf{R}$ (1987) Electrical tuning of hair cells in the inner ear. Trends Neurosci 10:421-425.

Flock $\AA$, Cheung HC (1977) Actin filaments in sensory cells of inner ear receptor cells. J Cell Biol 75:339-345.

Flock $\AA$, Flock B, Murray E (1977) Studies on the sensory hairs of receptor cells in the inner ear. Acta Otolaryngol (Stockh) 83:85-91.

Flock $\AA$, Flock B, Ulfendahl M (1986) Mechanisms of movement in outer hair cells and a possible structural basis. Arch Otorhinolaryngol 243:83-90.

Forge A (1991) Structural features of the lateral walls in mammalian cochlear outer hair cells. Cell Tissue Res 265:473-483.

Gold T (1948) Hearing. The physical hasis of the action of the cochlea. Proc R Soc Edinburgh [Biol Sci] 135:492-498.

Harrison RV, Evans EF (1979) Cochlear fibre responses in guinea pigs with well defined cochlear lesions. Scand Audiol [Suppl] 9:83-92.

Holley MC (1991) High frequency force generation in outer hair cells from the mammalian cochlea. Bioessays 13:115-120. 
Holley MC, Ashmore JF (1988) On the mechanism of a high-frequency force generator in outer hair cells isolated from the guinea pig cochlea. Proc R Soc Lond [Biol] 232:413-429.

Housley GD, Ashmore JF (1992) Ionic currents of outer hair cells isolated from the guinea-pig cochlea. J Physiol (Lond) 448:73-98.

Hubbard AE, Mountain DC (1983) Alternating current delivered into the scala media alters sound pressure at the eardrum. Science 222: 510-512.

Hudspeth AJ (1989) How the ear's works work. Nature 341:397-404.

Hudspeth AJ, Corey DP (1977) Sensitivity, polarity and conductance change in the response of vertebrate hair cells to controlled mechanical stimuli. Proc Natl Acad Sci USA 74:2407-2411.

Johnstone BM, Patuzzi R, Yates GK (1986) Basilar membrane measurements and the traveling wave. Hearing Res 22:147-153.

Kachar B, Brownell WE, Altschuler WE, Fex J (1986) Electrokinetic shape changes of cochlear outer hair cells. Nature 322:365-368.

Kemp DT (1978) Stimulated acoustic emissions from the human auditory system. J Acoust Soc Am 64:1386-1391.

Khanna SM, Flock $\AA$, Ulfendahl M (1989) Comparison of the tuning of outer hair cells and the basilar membrane in the isolated cochlea. Acta Otolaryngol [Suppl] (Stockh) 467:151-156.

Kim DO (1986) Active and nonlinear cochlear biomechanics and the role of the outer hair cell subsystem in the mammalian auditory system. Hearing Res 22:105-114.

Kolston PJ, de Boer E, Viergever MA, Smoorenburg GF (1990) What type of force does the cochlear amplifier produce? J. Acoust Soc Am 88:1794-1801.

Kros CJ, Crawford AC (1990) Potassium currents in inner hair cells isolated from the guinea-pig cochlea. J Physiol (Lond) 421:263-291.

Lighthill J (1991) Biomechanics of hearing sensitivity. J Vibr Acoust 113:1-13.

Lim DJ (1980) Cochlear anatomy related to cochlear micromechanics. A review. J Acoust Soc Am 67:1686-1695.

Mountain DC (1980) Changes in endolymphatic potential and crossed olivocochlear bundle stimulation alter cochlear mechanics. Science 251:71-72.

Mountain DC, Hubbard AL, McMullen TA (1983) Electromechanical processes in the cochlea. In: Mechanics of hearing (de Boer E, Viergever ME, eds), pp 119-126. Delft: Nijhoff.

Neely ST, Kim DO (1983) An active cochlear model showing sharp tuning and high sensitivity. Hearing Res 9:123-130.

Palmer AR, Russell IJ (1986) Phase-locking in the cochlear nerve of the guinea pig and its relation to the receptor potentials of inner hair cells. Hearing Res 24:1-15.

Pickles JO, Comis SD, Osborne MP (1984) Cross-links between stereocilia in the guinea pig organ of Corti, and their possible relation to sensory transduction. Hearing Res 15:103-112.

Rhode WS (1971) Observation of the vibration of the basilar membrane in squirrel monkeys using the Mössbauer technique. J Acoust Soc Am 49:1218-1231.

Rhode WS (1973) An investigation of postmortem cochlear mechanics using the Mössbauer effect. In: Basic mechanisms of hearing (Møller AR, ed), pp 49-67. New York: Academic.
Ruggero MA, Rich N (1991) Application of a commercially-manufactured Doppler-shift laser velocimeter to the measurement of basilar-membrane motion. Hearing Res 51:215-230.

Russell IJ, Richardson GP (1987) The morphology and physiology of hair cells in organotypic cultures of the mouse cochlea. Hearing Res 31:9-24.

Ryan AF, Dallos P (1975) Absence of cochlear outer hair cells: effect on behavioural auditory threshold. Nature 253:44-46.

Santos-Sacchi J (1989) Asymmetry in voltage-dependent movements of isolated outer hair cells from the organ of Corti. J Neurosci 9:2954 2962.

Santos-Sacchi J (1991) Reversible inhibition of voltage-dependent outer hair cell motility and capacitance. J Neurosci 11:3096-3110.

Santos-Sacchi J, Dilger DP (1988) Whole cell currents and mechanical responses of isolated outer hair cells. Hearing Res 35:143-150.

Sellick PM, Patuzzi RB, Johnstone BM (1982) Measurement of basilar membrane motion in the guinea-pig using the Mössbauer technique. J Acoust Soc Am 72:131-141.

Spoendlin H (1970) Structural basis of peripheral frequency analysis. In: Frequency analysis and periodicity detection in hearing (Plomp R, Smoorenburg GF, eds), pp 2-36. Leiden: Sijthoff.

Steele CR, Taber LA (1981) Three-dimensional model calculations for guinea-pig cochlea. J Acoust Soc Am 69:1107-1111.

Tilney LG, DeRosier DJ, Mulroy RB (1980) The organization of actin filaments in the stereocilia of cochlear hair cells. J Cell Biol 86:244259.

Voldrich L (1978) Mechanical properties of the basilar membrane. Acta Otolaryngol (Stockh) 86:331-335.

von Békésy G (1960) Experiments in hearing. New York: McGraw Hill.

Warr WB, Guinan JJ Jr (1979) Efferent innervation of the organ of Corti: two separate systems. Brain Res 173:152-155.

Weiss TF, Peake WT, Ling A, Holton T (1978) Which structures determine frequency selectivity and tonotopic organization of vertebrate cochlear nerve fibers? Evidence from the alligator lizard. In: Evoked electrical activity of the auditory nervous system (Naunton R, Fernàndez, eds), pp 91-112. New York: Academic.

Wever EG (1949) Theory of hearing. New York: Wiley.

Wilson JP (1980) Evidence for a cochlear origin for acoustic re-emissions, threshold fine-structure and tonal tinnitus. Hearing Res 2:233252.

Zenner HP (1986) Motile responses in outer hair cells. Hearing Res 22:83-90.

Zenner HP, Zimmerman U, Schmitt U (1985) Reversible contraction of isolated mammalian cochlear hair cells. Hearing Res 18:127-133.

Zwicker E (1979) A model describing nonlinearities in hearing by active processes with saturation at $40 \mathrm{~dB}$. Biol Cybern 35:243-250.

Zwislocki JJ (1980) Five decades of research on cochlear mechanics. J Acoust Soc Am 67:1679-1685.

Zwislocki JJ (1990) Active cochlear feedback: required structure and response phase. In: Mechanics and biophysics of hearing (Dallos $P$, Geisler CD, Matthews JW, Ruggero MA, Steele CR, eds), pp 114 120. New York: Springer. 